\title{
Triple play quality of service analysis in IP network
}

\author{
Agus Sri Budi Cahyono ${ }^{1 *}$, Gunawan Wibisono ${ }^{2}$ \\ 1, 2 Department of Electrical Engineering, University of Indonesia, Depok, Indonesia
}

\author{
Index Terms \\ Bandwidth Starvation \\ Traffic Policing \\ WFQ \\ LLQ \\ RED \\ Diffserv
}

Received: 26 July 2016

Accepted: 26 August 2016

Published: 25 October 2016

\begin{abstract}
Bandwidth starvation is an important aspect to consider when deploying Triple Play Quality of Service (QoS) in Internet Protocol (IP) network. How to guarantee each class of QoS running smoothly with enough bandwidth when facing congested network. Traffic policing technique has been proposed by author to ensure voice traffic is separated from other class instead of using Low Latency Queuing (LLQ) of Weighted Fair Queuing (WFQ). This paper addresses the effect of traffic policing and Random Early Detection (RED) to quality of service in term of delay, jitter and packet loss. The result show traffic policing with combination using RED is promised each class of WFQ gets enough bandwidth and avoid bandwidth starvation. Simulation results show end to end delay for voice 0.02 second, voice jitter 0.00025 second,video delay 0.05 second,video jitter 0.0005 . These paramaters accepted ITU-Y1541 standard. For queuingdelay paramater the result is 0.02 second.
\end{abstract}

(C) 2016 The Author(s). Published by TAF Publishing.

\section{INTRODUCTION}

Quality of Service (QoS) is an important aspect of triple play service implementation. Customer value expectation is depend on how QoS is applied. Problems arise related with QoS term such as low throughput, dropped packet, errors, latency, jitter and out of delivery. Diffserv is the one of QoS technique beside Intserv/Resource Reservation Protocol (RSVP) and IEEE 802.1/Q standard. Queuing technique in diffserv is main aspect to analyze related to the performance of triple play services. Previous research used Weighted Fair Queuing (WFQ) with Low Latency Queuing (LLQ) for voice, video and data class with Diffserv Service Code Point (DSCP) as traffic marking[1]. Priority Queuing with implementation as LLQ is used to service video and voice in that paper.

The result ; bandwidth starvation is occured when video class is using LLQ. This paper conclude that LLQ can only be implemented in voice traffic because voice trafic is very small. When huge voice traffic is transmitted ; bandwidth starvation is occured again.

\footnotetext{
* Corresponding author:Agus Sri Budi Cahyono

†Email: agus.sri@ui.ac.id
}

Random Early Detection (RED) is one of the dropping technique to smooth QoS delivery[2]. When network congestion is experienced, packet discard can't be avoided but can be controlled by several dropping techniques. Traffic policing or traffic shapping is part of how to improve QoS performance. Single rate traffic policing can be used for controll and manage traffic for QoS[3]. In this paper single rate traffic policing technique is implemented to separated voice from WFQ instead of using LLQ. With certain bandwidth allocation for voice and other class, results show that each class running smoothly without LLQ with better delay and jitter value.

After that RED is used to improve all class to control packet discard because of congestion. This paper is organized as follow. Section II describe the proposed model for traffic policing technique using single rate traffic policing in WFQ and RED traffic control for dropping packet mechanism. Simulation setup is presented in Section III. And simulation results are shown in Section IV. Finally the conclusions are presented in section $\mathrm{V}$. 


\section{PACKET HANDLING}

\section{A. Traffic Policing Proposed Model}

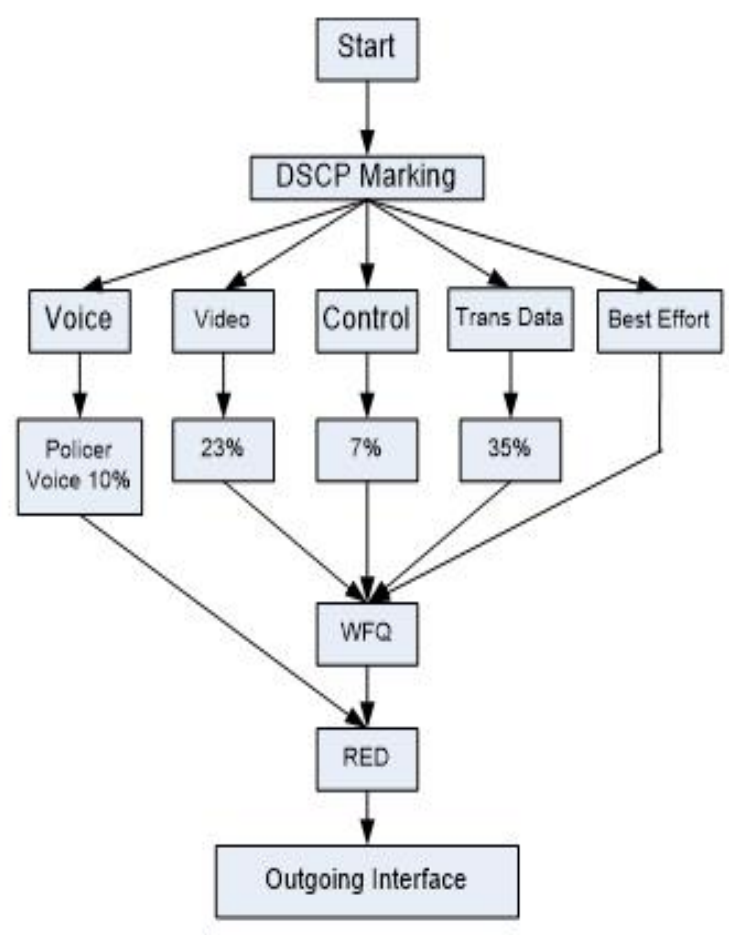

Fig. 1. Traffic policing proposed model

Figure 1 explain that the policing model use five class of marking; voice, video, control, trans data, and best effort. The model is preferred to consider all of the traffic classification is defined to support end to end QoS. Triple play use voice, video, and data which are the part of end to end Qos also, thus triple play must consider other class to define bandwidth allocation for policing. Best effort class is not policing to spesified bandwidth, so best effort traffic can use any available bandwidth from other class. Voice class is policed and not include to WFQ scheduler to increase voice performance to make voice still priority. The rest of the model used RED to manage packet drop. Control class used for routing and signalling traffic to run. Trans data and best effort are detail classification of data class to ensure different priority of data. Which is transactional data has higher priority tha best effort data. DSCP is used for marking traffic. Voice use EF, Video use AF41, Control use CS3, Trans data (AF21) and Best Effort use Default Forwarding (DF). The bandwidth value allocation for voice and for WFQ using RFC 3246, 2597, 2474 and 3662 [3].

\section{B. Single Rate Traffic Policing for Voice}

Single rate traffic policing has been used to separate voice traffic from others. Its done with defined subrate connection to flow from maximum access rate. There are three parameters related to this policing;

- Commited Information Rate (CIR)

- CIR is average maximum for voice traffic to flow in access rate $\mathrm{CIR}<$ Access Rate

- Commited Burst Size (Bc)

- Traffic excess CIR is defined with Bc for burst traffic.

- Excessive Burst Size (Be)

- Traffic excess Bc is defined with Be for excessive burst traffic.

With CIR $=\mathrm{Bc} / \mathrm{Tc}$, Tc is time cycle or period. Algoritm for single rate traffic policing shown in Figure 2. Voice traffic to be transmitted is ruled with CIR $10 \%$ from congestion link rate with $\mathrm{Bc}=\mathrm{CIR} / 8$. The excess traffic is drop. Three rule for voice traffic is conform, exceed and violate. Conform means traffic is agree with rule CIR and Bc. Otherwise exceed and violate are use to rule excessice traffic. With this algoritm voice traffic can flow with $10 \%$ bandwith value when congestion happen. The $\mathrm{Bc}$ value with $\mathrm{CIR} / 8$ is choosen for standard implementation to make voice rule run with $10 \%$.

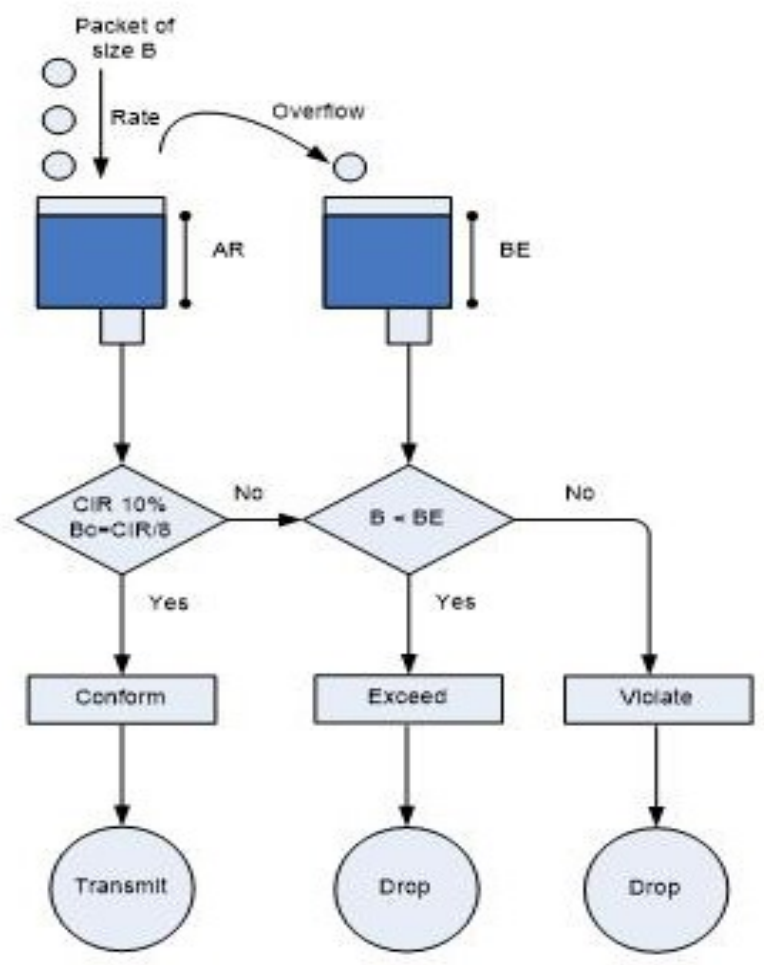

Fig. 2. Single rate traffic policing 


\section{RED Traffic Drop Policer}

RED operates by computing average queue length and compare to minimum and maximum threshold then make a packet drop decision [2]. When the queue size is increasing, incoming packets will be dropped with a higher probability. Finally, if average queue length is higher than the queue size threshold then all incoming packet will be dropped completely [2] as shown in Figure 3.

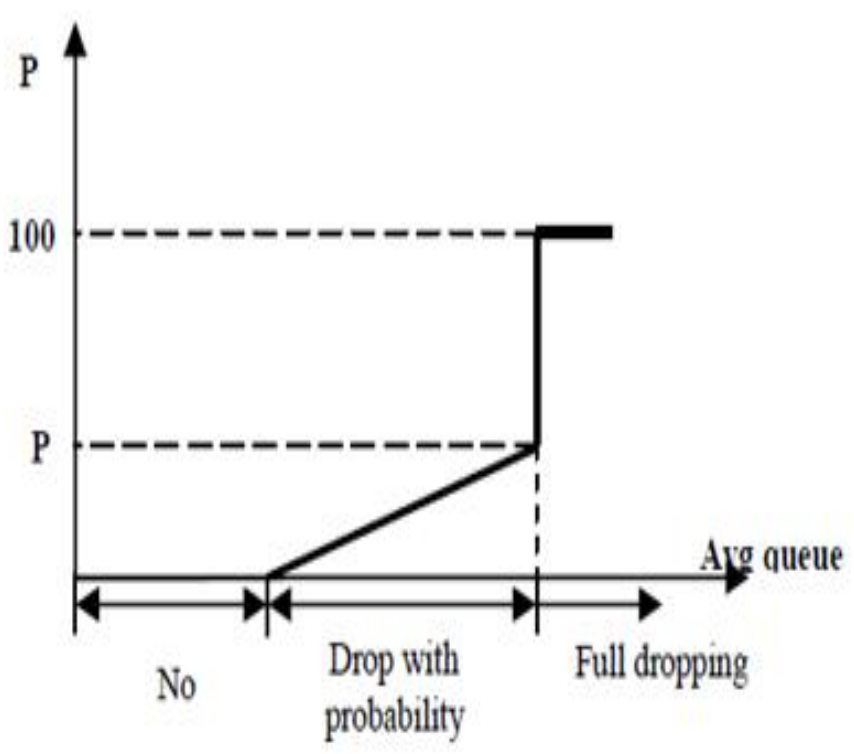

Fig. 3 . RED scheme [2]

RED algorithm based on Figure 4 is applied for all incoming traffic from voice policing and from WFQ based. All traffic is calculated for average packet and calculate for packet dropping probability to decide drop or enqueue the packet. RED parameter has been iterated to get best value in order to make all traffic run with best drop scheme. Minimum threshold value is set to 1 packet, maximum threshold value is set to 2 packet, mark probability denominator is set to $1 / 10$ and exponential weighting constant is set to 9 .

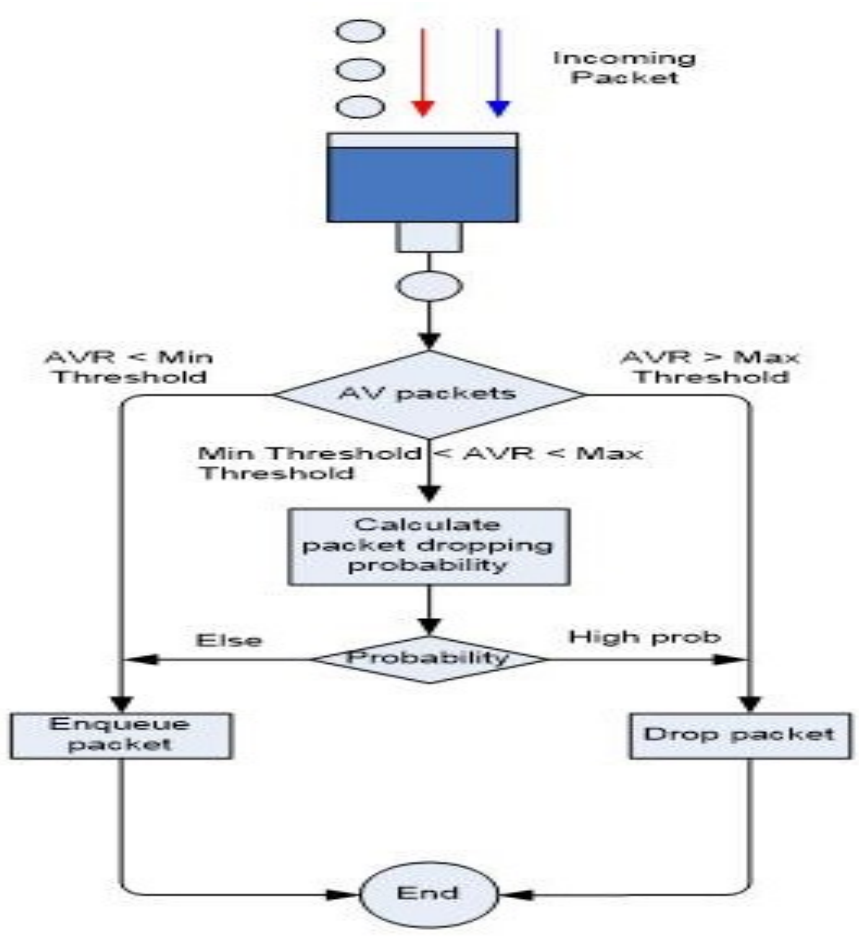

Fig. 4. RED algoritm

\section{OPNET SIMULATION MODEL}

Simulation of Triple Play architechture is based on OPNET tool[8]. The network topology use router, workstation and server. All network component are connected by DS3 link. QoS function set up by IP QoS Attribute Definition and local router QoS configuration [3], [10]. Link from end router to destination is observed and modified to simulate congestion link by change DS3 data rate to $10 \mathrm{Mbps}$. Explicit demand traffic used to simulate the demand from each class. Detail marking, policing and CBWFQ bandwidth allocation are detailed below:

TABLE 1

TABLE I. DETAIL MODEL PARAMETER POLICED

\begin{tabular}{llllll}
\hline \hline Class & Traffic Source Size & Traffic Source PDF & DSCP Marking & CB-WFQ Bandwidth & Policy \\
\hline Voice & T1 $(1.5 \mathrm{Mbps})$ & Exponential & EF & - & CIR $=10 \% \mathrm{Mbps} \mathrm{Bc}=\mathrm{CIR} / 8 \mathrm{Mbps}$ \\
Video & $8 \mathrm{Mbps}$ & Exponential & AF41 & $23 \%$ & - \\
Control & $\mathrm{T} 1(1.5 \mathrm{Mbps})$ & Exponential & $\mathrm{CS} 3$ & $7 \%$ & - \\
Trans Data & $\mathrm{T} 1(1.5 \mathrm{Mbps})$ & Exponential & AF21 & $35 \%$ & - \\
\hline \hline
\end{tabular}


The aim of simulation is to study the effect of traffic policing instead of using LLQ. Traffic policing is applied to voice traffic only with defined average traffic rate and burst size. For Video, Control, Trans Data, traffic is defined using spesific WFQ bandwidth allocation. Best Effort traffic does not policed in order to make bandwidth efficiency allocation. The network diagram is shown below:

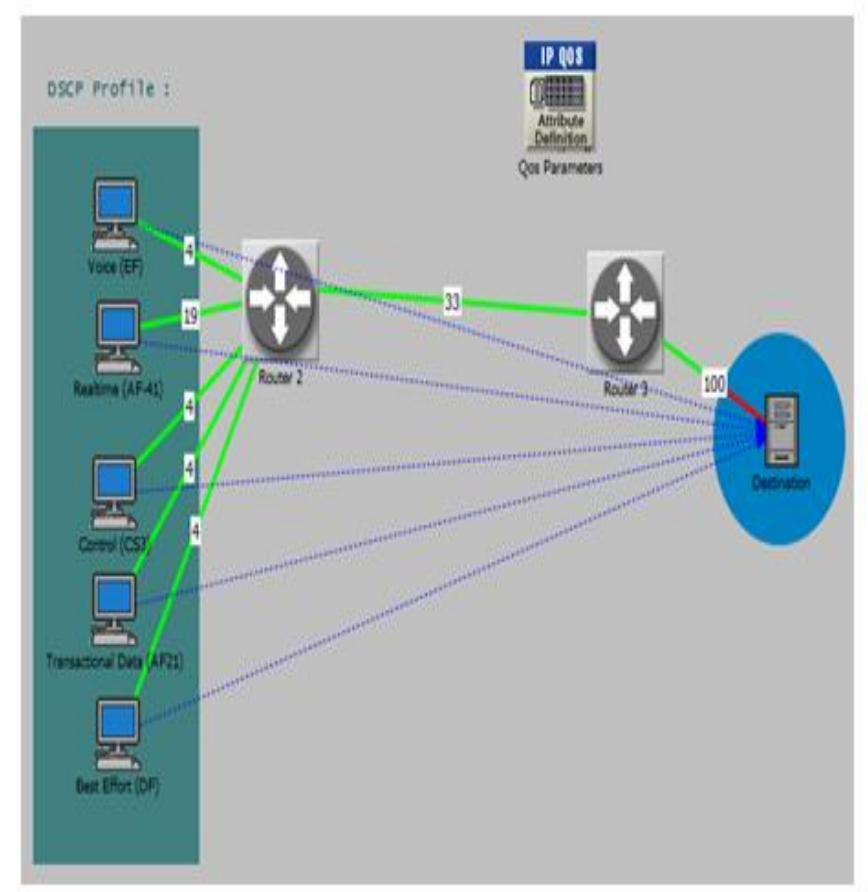

Fig. 5. OPNET network diagram

For comparison from the previous work, four step have been used for simulation. First step is represent the previous work using WFQ-LLQ. Second step is using CBWFQ-LLQ. Third step is using CBWFQ with voice policed and the fourth step is CBWFQ with voice policed and RED.

\section{SIMULATION RESULTS}

With voice traffic send according to Figure 6, simulation results show that voice traffic received run with the defined rule which the rule is voice must run with $10 \%$ from congestion link rate $10 \mathrm{Mbps}$ which is $1 \mathrm{Mbps}$ as shown in Figure 7. Its show that policer can manage bandwidth allocation for voice traffic, therefore can avoid bandwidth starvation for other class. Number in circle from 1 to 4 is represent steps have been used to compare from previous step and work. From Figure 7 shows that step 3 and step
4 which use voice policed is can manage voice to run and flows using specific rate. However with LLQ mode in step 1 and 2 show that all traffic send are received with same value of rate. From Figure 7 because voice used $1 \mathrm{Mbps}$ from congestion link so other class traffic could use $9 \mathrm{Mbps}$ from remaining bandwidth.

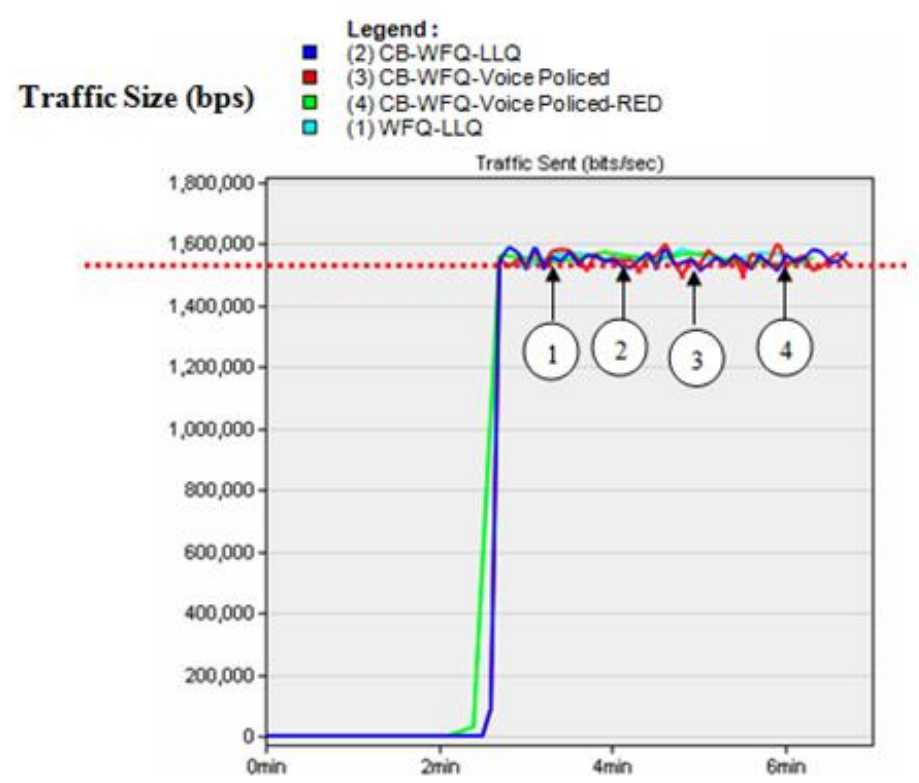

Fig. 6. Voice traffic send

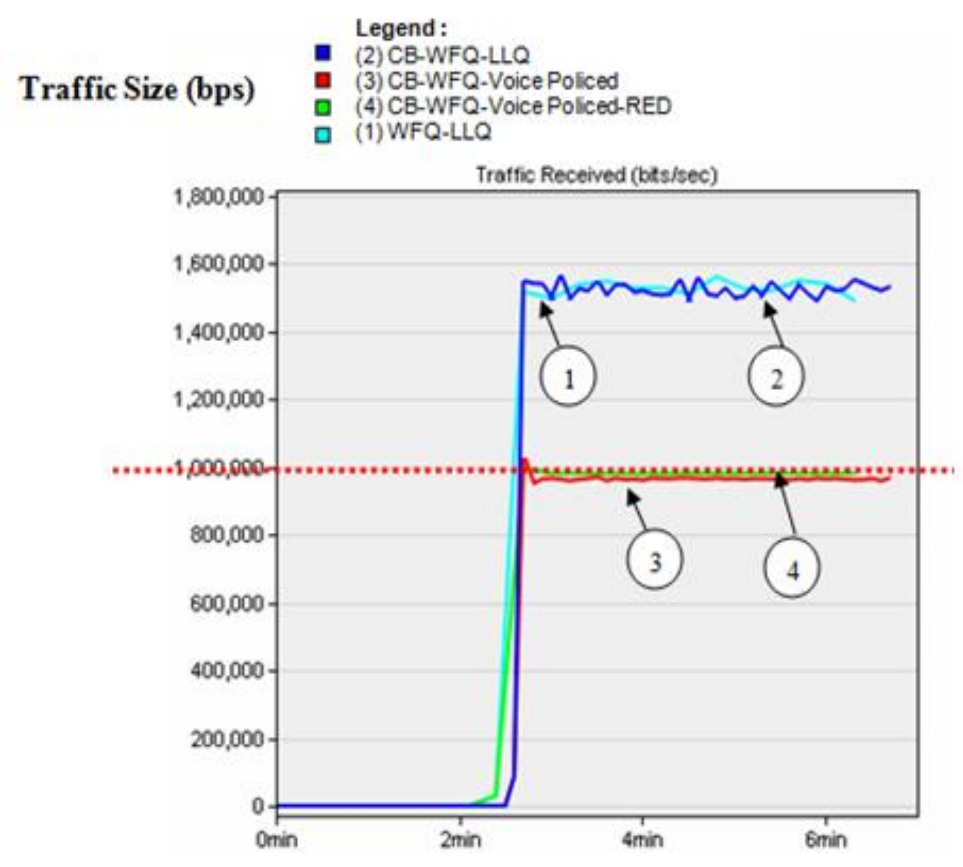

Fig. 7 . Voice traffic received 
End to end delay value from Figure 8 for step 4 shows about 0.02 second, which is acceptable for voice regarding ITU-Y1541 recommendation $<0.15$ second. The red line across the figure represent the ITU-Y1541 recommendation for voice end to end delay. For jitter parameter refer to Figure 9 shows that voice jitter also acceptable with ITUY1541 recommendation. Separate voice traffic from other traffic and using RED for control traffic drop is the key. RED control how traffic dropping occured thus can reduce delay and jitter value. RED technique used for improve the end to end delay caused of dropping packet. With RED all class are improved in end to end delay parameter. It is because RED can control how packet dropped during congestion condition.

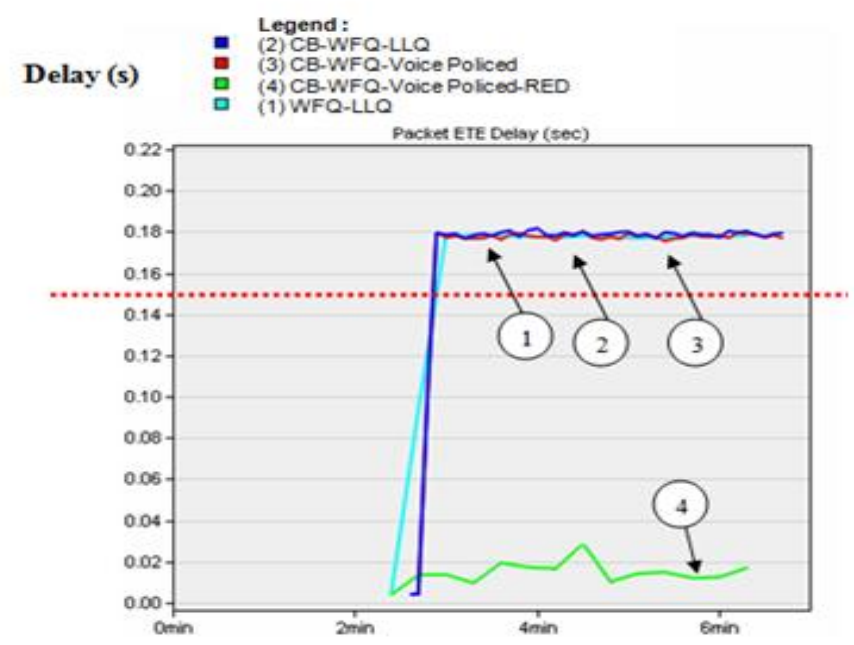

Fig. 8. Voice E2E delay

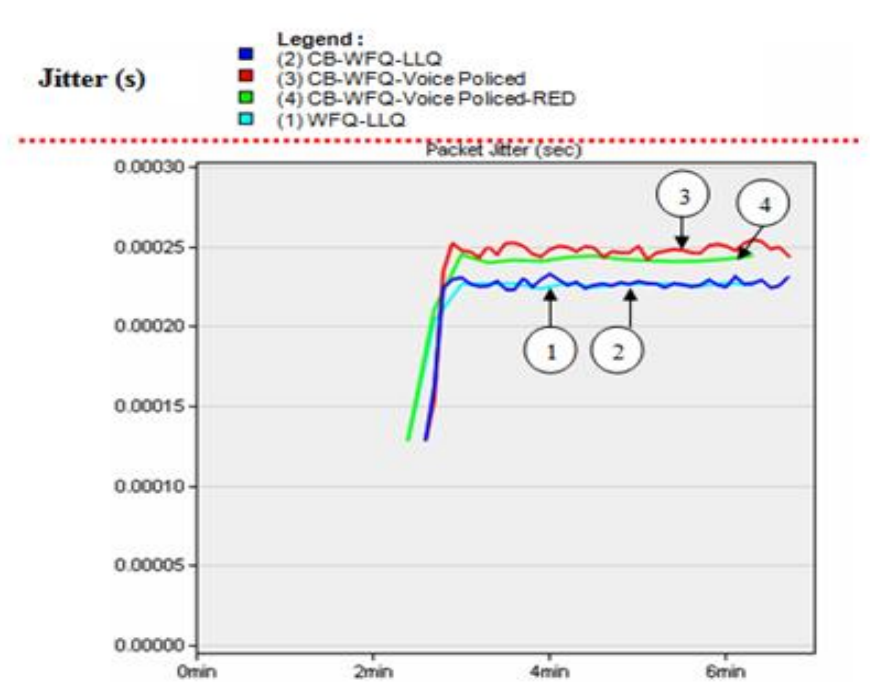

Fig. 9 . Voice jitter
Figure 10 shows video E2E delay value for step 4 with 0.03 second, which is accepted in ITU-Y1541 recommendation $<0.3$ second. Also Figure 11 shows video jitter parameter is accepted with ITU-Y1541 recommendation < 0.04 second.

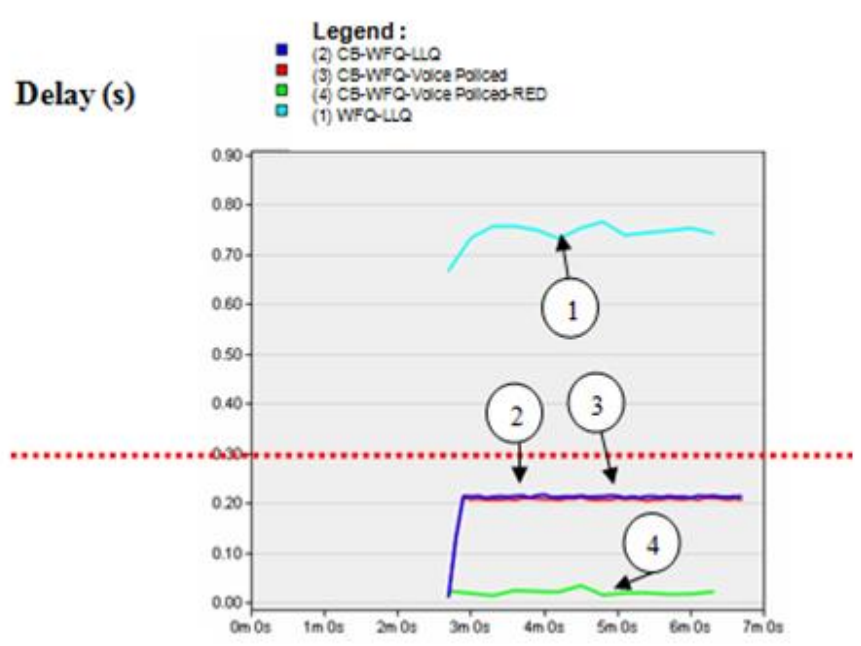

Fig. 10. Voice E2E delay

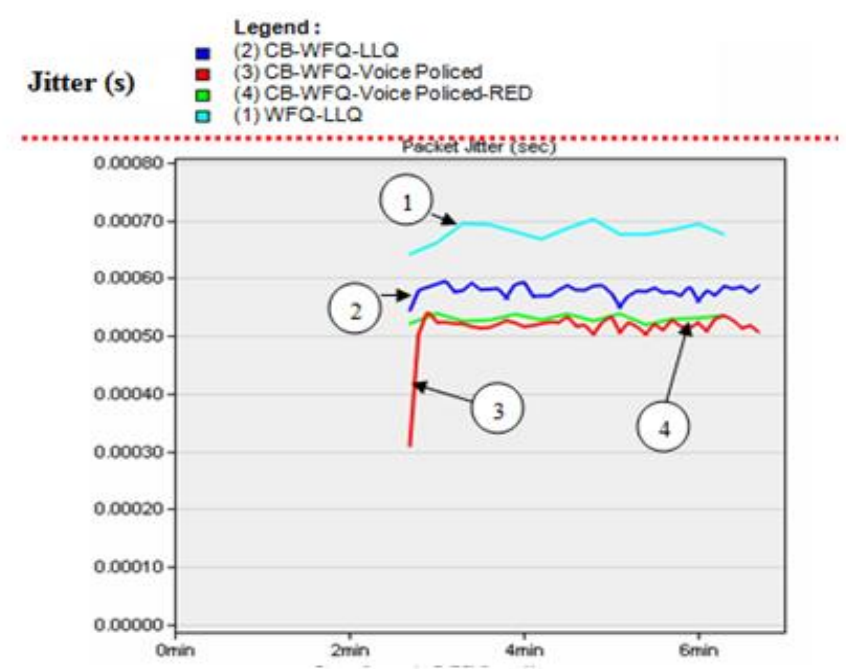

Fig. 11. Voice jitter

Result summary of P2P utilization gives in Figure 12. It can be shown that link utilization not full to $100 \%$ when congestion occured, so this technique can make link utilization more efficient. Also queuing delay when congestin occured is shown in Figure 13. Queuing delay for step 4 is dropped to previous step. Queuing delay is very important aspect to consider when congestion occured. 


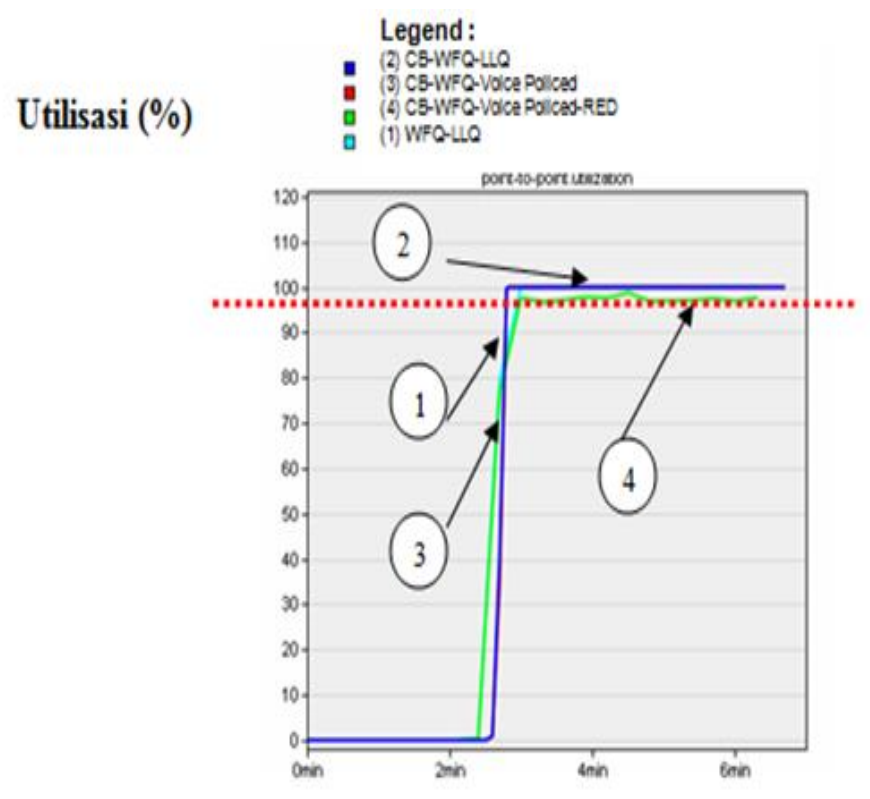

Fig. 12 . Point to point utilization

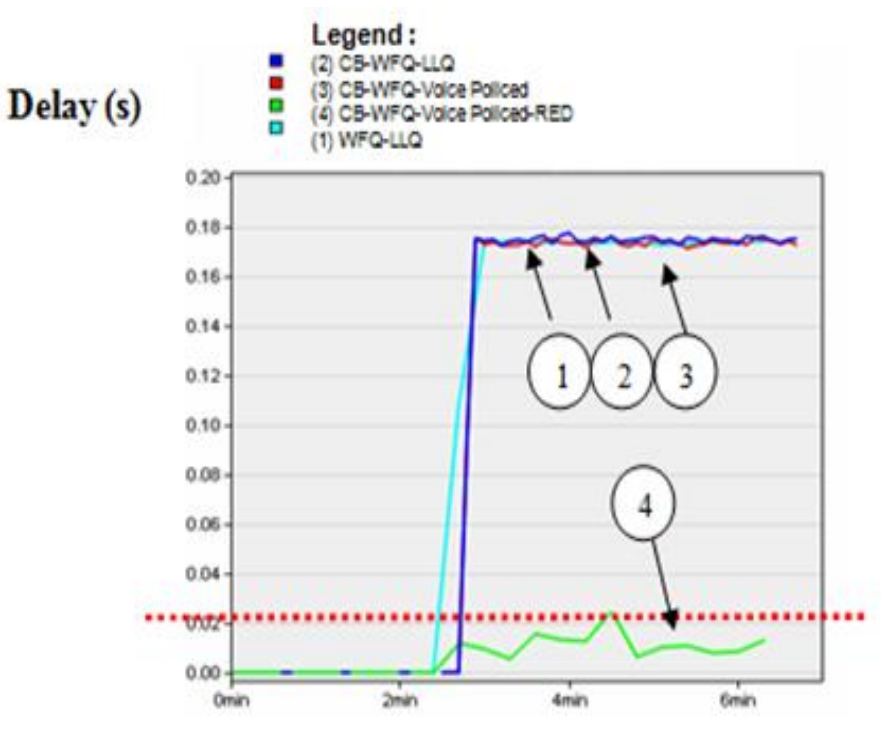

Fig. 13. Point to point queuing delay

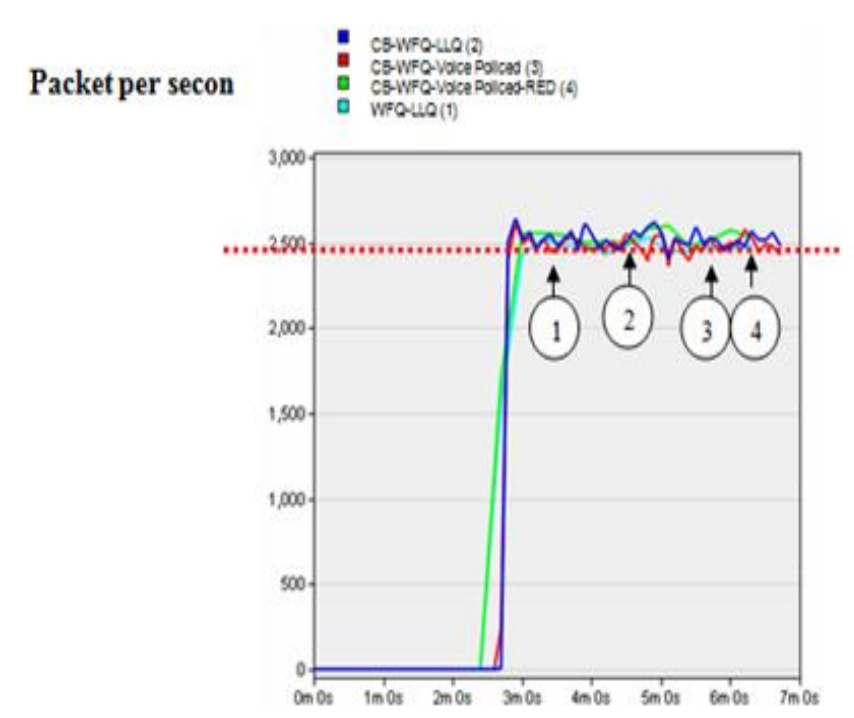

Fig. 14. IP Traffic drop

\section{CONCLUSION}

Traffic policing can ensure that traffic can be delivered with certain bandwidth without starvation. It can be applied on voice traffic therefore to make voice still prioritized. With traffic policing jitter perfomance also improved. RED used to improve end to end delay regards traffic dropped. With RED traffic drop is controlled and managed. Thus traffic policing for voice traffic with combination with RED is best choice for improve QoS for triple play service instead of using LLQ.

\section{ACKNOWLEDGMENT}

This work was fully supported by PT Telekomunikasi Indonesia as a honour to held research on data services to improve Quality of Service.

\section{REFERENCES}

[1] S. Badr, F. Bayoumi and G. Darwesh "QoS adaptation in real time systems based on CBWFQ" in 28th IEEE National Radio Science Conference (NRSC), 2011, pp. 1-8. DOI: 10.1109/NRSC.2011.5873626

[2] G. Su, T. O. Pham and S. He, "QoS guarantee for IPTV using low latency queuing with various dropping schemes," in International Conference on Systems and Informatics (ICSAI), 2012, pp. 1551-1555. D0I: 10.1109/icsai.2012.6223334

[3] T. Szigeti, C. Hattingh, R. Barton, K. Briley JR, End To End QoS Network Design. Indianapolis, IN: Cisco Press, 2013.

[4] S. M. Eljack, S. B. Abdelkarim, "Effect of the interior gateway routing protocols in the multiprotocol label switching networks a simulated comprison between IS-IS \& OSPF routing protocols," in International Conference on Computing, Electrical and Electronics Engineering (ICCEEE), 2013, pp. 565-570. DOI: 10.1109/ICCEEE.2013.6634002 
[5] R. Yunos, S. A. Ahmad, N. M. Noor, R. M. Saidi, Z. Zainol, "Analysis of routing protocols of VoIP VPN over MPLS network," in IEEE Conference on Systems, Process \& Control (ICSPC), 2013, pp. 139-143. DOI: 10.1109/SPC.2013.6735120

[6] H. Hass, "Queuing methods from FIFO to CB-WFQ/PQ," 2009.

[7] A. Mobiu and R. Hartanto, "Analysis quality of service from Internet protocol television (IPTV) service," International Journal of Informatics and Communication Technology (IJ-ICT), vol. 1, no. 2, 100-108, 2012.

[8] H. M. Asif and E. M. El-Alfy, "Simulation-based performance comparison of queueing disciplines for differentiated services using OPNET,"

[8] M. F. de Castro, D. Garti, A. M'hamed and M. Oliveira, "Comparing application performance on distinct ip packet scheduling configurations," in Proceding of 21st Brazilian Symposium on Computer Networks (SBRC), 2003, pp. 441-54.

[10] H. A. Mohammed, A. H. Ali and H. J. Mohammed, "The affects of different queuing algorithms within the router on QoS VoIP application using OPNET," 2013.

— This article does not have any appendix. — 